University of Louisville ThinkIR: The University of Louisville's Institutional Repository

Electronic Theses and Dissertations

$5-2019$

\title{
A study on radiative pion capture as a source of background in the search for muon to electron conversion at the Mu2e experiment.
}

Joseph Leibson

University of Louisville

Follow this and additional works at: https://ir.library.louisville.edu/etd

Part of the Elementary Particles and Fields and String Theory Commons

\section{Recommended Citation}

Leibson, Joseph, "A study on radiative pion capture as a source of background in the search for muon to electron conversion at the Mu2e experiment." (2019). Electronic Theses and Dissertations. Paper 3170.

https://doi.org/10.18297/etd/3170

This Master's Thesis is brought to you for free and open access by ThinkIR: The University of Louisville's Institutional Repository. It has been accepted for inclusion in Electronic Theses and Dissertations by an authorized administrator of ThinkIR: The University of Louisville's Institutional Repository. This title appears here courtesy of the author, who has retained all other copyrights. For more information, please contact thinkir@louisville.edu. 


\title{
A STUDY ON RADIATIVE PION CAPTURE AS A SOURCE OF BACKGROUND IN THE SEARCH FOR MUON TO ELECTRON CONVERSION AT THE MU2E EXPERIMENT
}

By

Joseph Leibson

B.S. Physics, University of Louisville, 2016

\author{
A Thesis \\ Submitted to the Faculty of the \\ College of Arts and Sciences of the University of Louisville \\ In Partial Fulfillment of the Requirements \\ for the Degree of \\ Master of Science \\ in Physics
}

Department of Physics and Astronomy

University of Louisville

Louisville, Kentucky

May 2019 
Copyright 2019 by Joseph Leibson

All rights reserved 



\title{
A STUDY ON RADIATIVE PION CAPTURE AS A SOURCE OF BACKGROUND IN THE SEARCH FOR MUON TO ELECTRON CONVERSION AT THE MU2E EXPERIMENT
}

\author{
by \\ Joe Leibson \\ B.S. Physics, University of Louisville, 2016
}

A Thesis Approved On

April 16th, 2019

by the following Thesis Committee:

Thesis Director

David Brown

Christopher Davis

Jacob Wildstrom 


\section{ACKNOWLEDGEMENTS}

In the first semester of undergrad at University of Louisville, Dr. David Brown was my instructor for an introductory lab. He was present for our first lab session unlike other instructors who opted to send their TA. During this session, Dr. Brown explained how motion and pressure sensors in the lab function and giddily explained a unique phenomenon essential to these measurement devices. "Piezoelectric crystals!”, he calmly

exclaimed, going on to describe how objects (namely crystals) are able to translate pressure and acceleration into an electrical signal used to generate measurements. Both the content of that lecture and his genuine enthusiasm helped motivate me to switch majors from Mathematics to Physics, and for that I am grateful.

Today, I thank Dr. Brown for his consistent help during the process of this study. Without his help this paper would not have been possible. Beyond Dr. Brown, I thank the entire Department of Physics \& Astronomy for their continued help throughout my time at University of Louisville. Without this program I would not have the skills nor the understanding of physics needed to complete this project. Throughout my time in the High Energy Physics research group there were four peers who helped me along this journey: Jake Colston, Joseph Brock, Brent Mode, and Connor Cummings. Without these four, this study would have taken far longer. To each of you, thank you.

To my brothers Stuart and Charlie, thank you for your constant support throughout life, I wouldn't be myself without you two. To my parents, Deborah and Michael, I thank you for giving me the opportunity to follow this semi-absurd path into 
physics and mathematics. Although these subjects weren’t familiar to you, your support never faltered. To Seth, Zach, Russell, and Joe, I'm grateful for all that you've done for me over the years. Adventures and discussions with each of you have helped me maintain sanity more than you may realize. 


\begin{abstract}
A STUDY ON RADIATIVE PION CAPTURE AS A SOURCE OF BACKGROUND IN THE SEARCH FOR MUON TO ELECTRON CONVERSION AT THE MU2E EXPERIMENT
\end{abstract}

Joseph Leibson

April 16th, 2019

The following thesis will cover the process of radiative pion capture in the Mu2e experiment at Fermi National Accelerator Laboratory. Radiative pion capture (RPC) is a process that is able to mimic the signal of an electron spawned from the neutrino-less conversion of a muon. In this paper we will discuss the current Standard Model accepted by physicists, and discuss charged lepton flavor violation, which is the motivation behind Mu2e. This paper will cover the theory behind Mu2e and RPC, as well as the apparatus involved in the Mu2e experiment. Next, software and simulations used to carry out the RPC study will be explained in detail. Following this discussion, final results of this background study will be presented. These results indicate that the RPC process is estimated to have little effect on the Mu2e experiment throughout its lifetime. 


\section{TABLE OF CONTENTS}

\section{Page}

ACKNOWLEDGEMENTS

iii

ABSTRACT

LIST OF TABLES

$\mathrm{V}$

LIST OF FIGURES

ix

$\mathrm{X}$

\section{CHAPTER}

1 INTRODUCTION

1.1 From the Four Elements to Elementary Particles . . . . . . . . 1

1.2 Charged Lepton Flavor Violation . . . . . . . . . . . . . 5

1.3 Mu2e. . . . . . . . . . . . . . . 6

1.4 Radiative Pion Capture . . . . . . . . . . . . 8

2 THEORY

2.1 Supersymmetry . . . . . . . . . . . . . . 10

2.1 .1 The Hierarchy Problem . . . . . . . . . . . . . . 10

2.1.2 Minimal Supersymmetric Standard Model . . . . . . . . . . 12

2.2 External and Internal Conversion . . . . . . . . . . . . 13

3 THE MU2E COMPLEX

3.1 Overview of Mu2e . . . . . . . . . . . . . . . 15 
3.2 Radiative Pion Capture . . . . . . . . . . . . . . . . . . .

3.3 From Proton to Stopped Muon . . . . . . . . . . . . . 16

3.3.1 Proton Beamline . . . . . . . . . . . . . . 17

3.3.2 Production Solenoid . . . . . . . . . . . . . 19

3.3.3 Transport Solenoid and Collimator . . . . . . . . . . . . . 20

3.3.4 Detector Solenoid and Stopping Target . . . . . . . . . . . 21

3.4 The Detector Complex . . . . . . . . . . . . . . 22

3.4.1 Stopping Target Monitor . . . . . . . . . . . . . . . . 22

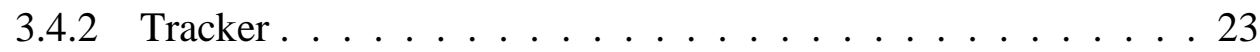

3.4.3 Electromagnetic Calorimeter . . . . . . . . . . . . 24

3.4.4 Cosmic Ray Veto . . . . . . . . . . . . . . . . . . 26

4 SOFTWARE \& SIMULATIONS

4.1 Software - Frameworks \& Packages . . . . . . . . . . . . . 29

4.1.1 Fermilab's art Framework . . . . . . . . . . . . . . . . 30

4.1 .2 Mu2e Offline . . . . . . . . . . . . . . 33

4.2 Simulations . . . . . . . . . . . . . . . 34

4.2.1 From POT to Stopped Pion . . . . . . . . . . . . . 35

4.2.2 Stopped Pion to Pair Production . . . . . . . . . . . 37

4.2.3 Tracker Hits, Reconstruction \& Analysis . . . . . . . . . . . 38

$5 \quad$ FINAL RESULTS

5.1 Final Results . . . . . . . . . . . . . 43

5.1.1 External \& Internal Conversion Results In-Time . . . . . . . . 43

5.1.2 External \& Internal Conversion Results Out-of-Time . . . . . 45

5.1 .3 Combined Final Results . . . . . . . . . . . . . . 46

5.2 Final Conclusions . . . . . . . . . . . . . 46 
REFERENCES 


\section{LIST OF TABLES}

\section{TABLE}

5.1 External Conversion Results . . . . . . . . . . . . . . . . . . 44

5.2 Internal Conversion Results . . . . . . . . . . . . . . . . . 45 


\section{LIST OF FIGURES}

\section{FIGURE}

1.1 Table of Elementary Particles . . . . . . . . . . . . . . . 3

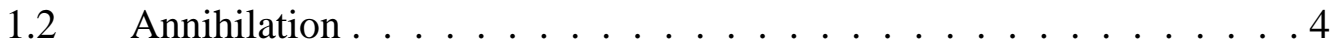

3.1 Proton Beamline . . . . . . . . . . . . . . . . 17

3.2 Proton Pulse . . . . . . . . . . . . . . . . . 18

3.3 Mu2e Apparatus. . . . . . . . . . . . . . . . . . 22

3.4 Tracker. . . . . . . . . . . . . . . . . . 24

3.5 Tracks of Conversion Electron. . . . . . . . . . . . . . . . 25

3.6 Mu2e Calorimeter. . . . . . . . . . . . . . . . . 26

3.7 Cosmic Ray Veto. . . . . . . . . . . . . . . . . . . 27

$4.1 \quad$ Example fcl File. . . . . . . . . . . . . . . . . . 31

4.2 Pion Stops in Z-Dimension. . . . . . . . . . . . . . . 38

4.3 Pion Stops in X-Dimension. . . . . . . . . . . . . . . . 40

$4.4 \quad$ Pion Stops in Y-Dimension. . . . . . . . . . . . . . . . . . . 41

4.5 Pion Stops in XY-plane. . . . . . . . . . . . . . . . . . 42 


\section{CHAPTER 1}

\section{INTRODUCTION}

\subsection{From the Four Elements to Elementary Particles}

The fundamental question, “What is matter?”, has puzzled thinkers, philosophers, and scientists since the beginning of humankind. Physical science may have begun with the Greek philosopher Empedocles (490-430 BCE) who identified the four elements of earth, air, fire and water. But it was another ancient Greek, Democritus (460-370 BCE), who came up with the concept of the atom as the smallest building block of matter. Fast forward more than two millennia to 1910, and Ernest Rutherford's gold foil experiment dispelled the notion that the atom was the most basic unit of matter. Protons, neutrons, and electrons provided proof that a more minute family of building blocks existed beyond the atom.

Interestingly, it was before Rutherford's breakthrough, in 1900, that German physicist Max Planck uncovered the basis of what we now know as Quantum Mechanics (QM). With the discovery of the Black Body Radiation spectrum and the assumed intrinsic quantization of light energy, QM was born. The existence of both QM experiments and Einstein's theory of Relativity challenged the tenets of scientific consensus at that time. Fortunately, these unintuitive cornerstones of QM extended our 
ability to study these newfound subatomic particles. Skipping ahead to the mid-1970’s, after numerous high energy physics (HEP) experiments, physicists used findings regarding sub-atomic particles and their interactions to build what is known as the Standard Model (SM). The basis of the SM wasn’t limited to what was already known about these subatomic particles; instead, predictions (such as the Higgs boson) were included into the framework. Since then, many of these supposed particles and interactions have been confirmed through experiment. However, certain predicted characteristics of these particles and their interactions have been disproven (i.e. neutrino oscillations). Due to these inconsistencies, we see a proliferation of alternate theories, one of which will be discussed later.

Much like the Periodic Table of Elements, the SM organizes matter into fundamental subatomic particles: six leptons, six quarks, and five bosons, which comprise all matter and interactions. Additionally, the SM includes information about the interactions that are able to occur among these particles. The three fundamental forces described by the SM are mediated via “force-carrying” particles known as bosons. In Quantum Mechanics, these bosons have integer spin values (i.e. $s=0,1$, 2, etc.) and follow Bose-Einstein statistics; hence, the name boson. Similarly, particles with a halfinteger spin (i.e. $s=1 / 2,3 / 2,5 / 2$, etc.) follow Fermi-Dirac statistics, and are referred to as fermions. We find that fermions create nearly all stable composite matter, and bosons account for all force mediators. This conclusion comes from the famous Pauli Exclusion Principle, which states that any two fermions are not allowed to occupy the same quantum state in the same space, whereas bosons are permitted to do so. This principle confirms our everyday observation that objects composed of rigid matter do not pass 
through one another, while matter in the form of bosons, like photons, are able to travel through one another (i.e. two lasers in the same space). Figure 1.1 details the fundamental particles and their respective quantum numbers.

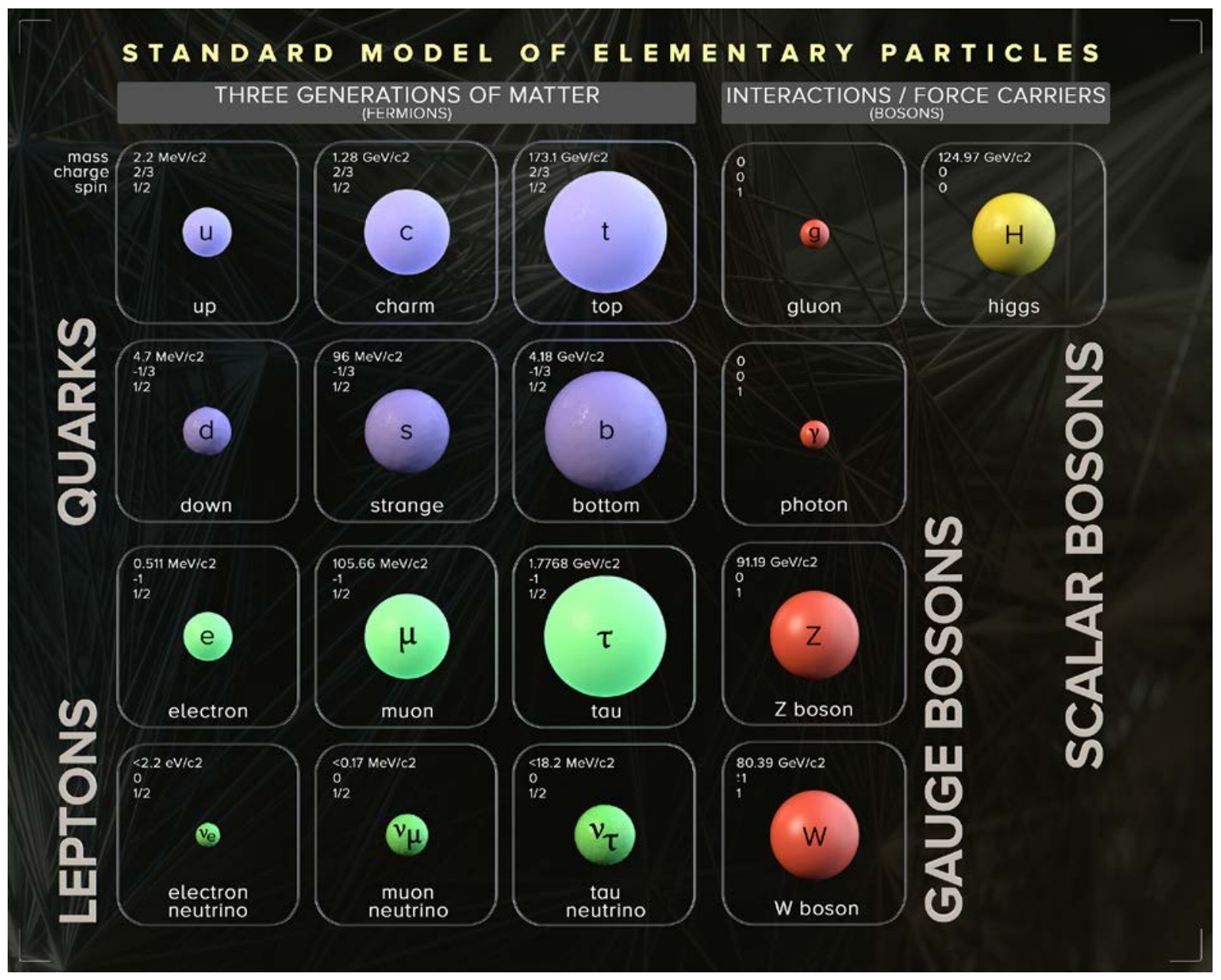

Figure 1.1: The Standard Model table of elementary particles (Resonant Language)

The three fundamental forces described by the SM include the strong force, the weak force, and the electromagnetic force (gravity is not described by the SM). These forces are mediated by the previously described bosons: gluons, the $\mathrm{W}$ and $\mathrm{Z}$ bosons, and photons. Gluons mediate the strong force, $\mathrm{W}$ and $\mathrm{Z}$ bosons mediate the weak force, and 
photons mediate the electromagnetic force. The strong force occurs between particles with color, or color charge, which is a quantum property of quarks as well as the mediating gluon. The weak force corresponds to the interaction between particles that contain flavor, which is a quantum property of leptons and quarks. Flavor will be detailed further in the next section of this paper. Lastly, the electromagnetic force occurs between all particles with electric charge. Though the Higgs boson doesn’t technically mediate a force, this is an appropriate time to mention it. The Higgs boson mediates what is known as the Higgs mechanism. The Higgs mechanism is the way in which particles obtain their mass, via interactions with the Higgs boson. Particles without mass (i.e. bosons such as photons and gluons) do not interact with the Higgs boson.

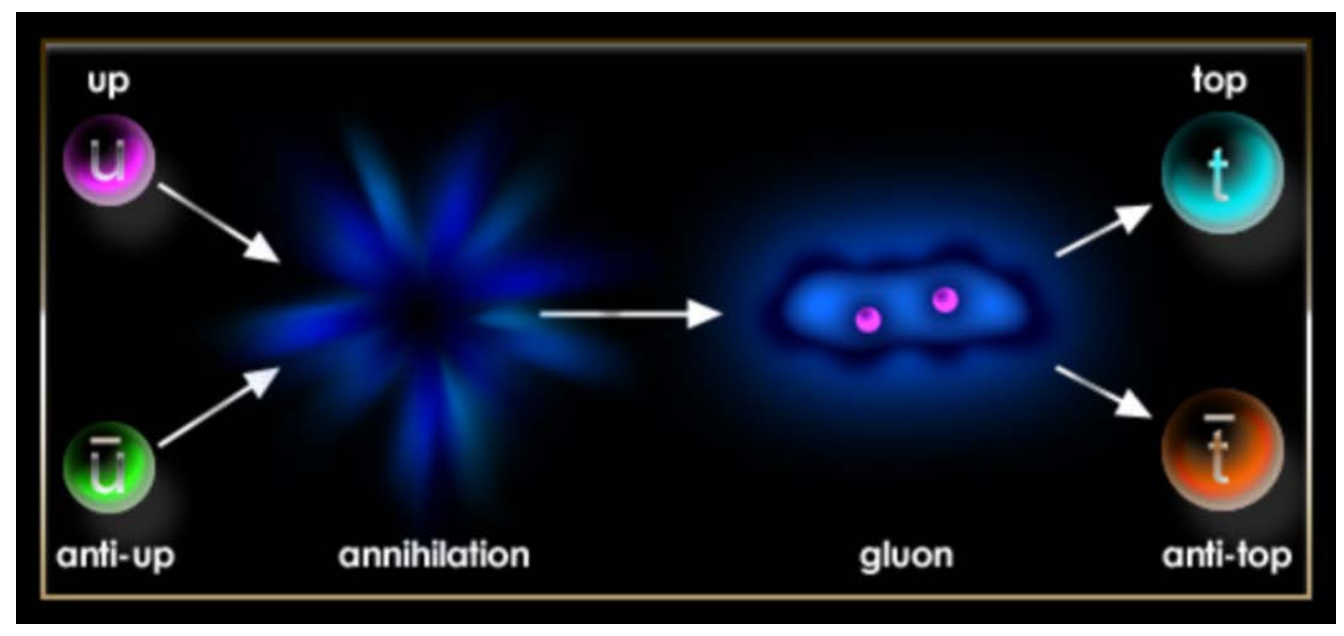

Figure 1.2: Particle annihilation of an up and anti-up quark (Particle Data Group)

To cap the overview of SM essentials, we must discuss the phenomenon of annihilation between particle and antiparticle. The collision between a fundamental particle and its corresponding antiparticle can result in their annihilation. This annihilation converts the initial mass energy into one or more bosons which may be either 
real or virtual. The resulting boson has energy of the initial system, and doesn't survive for long, due to Heisenberg's Uncertainty principle. In the case of a real boson, the input energy from the collision must equal the rest mass of a boson. If the input energy doesn’t equal the rest mass of a boson, then the result will be a virtual particle. Virtual particles instantly convert into a particle-antiparticle pair. Figure 1.2 is a schematic representing the annihilation of an up quark and anti-up quark pair. The result of this specific annihilation is a top quark and anti-top quark; however, as previously stated, these resultants represent one of many possibilities. The apparent reverse of annihilation, is known as a process called pair production. The most common form of pair production is when a photon is converted into an electron-positron pair, which occurs once the photon is close to an atomic nucleus. The reason why this process occurs near the nucleus in order to conserve the momentum of this interaction, otherwise the initial momentum would be zero and the resulting momentum would be nonzero. Both of these processes will prove to be relevant in the upcoming chapter.

\subsection{Charged Lepton Flavor Violation}

In the world of HEP, particle flavor is contained by the different types of fundamental particles involved in an interaction. Our SM predicts that flavor is a conserved quantity during any interaction. Quark flavor violation was observed in the nascent stages of HEP experiments before the SM, and was therefore never a part of the SM. Alternatively, lepton flavor violation (LFV) was included in the SM, but was not confirmed until 1998 when the Super-K experiment discovered neutrino oscillations 
(Nunokawa. et al). Though our current version of the SM is a solid basis for

understanding the subatomic nature of reality, the scientific community concedes that the SM does not provide a complete picture. Neutrino oscillations, proving the existence of LFV, illustrate a fault within the SM.

Despite having the proof of LFV for more than twenty years, charged lepton flavor violation (CLFV) has yet to be confirmed through experiment. In theories beyond the SM, like super-symmetry models, CLFV occurs at rates more measurable than those predicted with the current model (Ilakovac et al., 2013).

\subsection{Mu2e}

Mu2e is an abbreviation of "muon to electron". This experiment seeks to observe the coherent conversion of a muon to an electron. The natural decay for a muon yields an electron and two neutrinos (an anti-electron neutrino and muon neutrino). The antielectron neutrino has an electron flavor of negative one; therefore the electron flavor on the right hand side of the interaction is zero, mirroring the left hand side. We also see that the muon flavor on each side of the interaction is one. Therefore, flavor is conserved in this interaction. However, in the case of Mu2e, we see an imbalance as we have a muon flavor of one on the left hand side and zero on the right hand side. Below are the interactions for free-muon decay and Mu2e. The top is an interaction involving free decay and the bottom equation is the Mu2e conversion, both in the presence of an Aluminium nucleus: 


$$
\begin{gathered}
\mu^{-}+(A, Z) \rightarrow(A, Z)+e^{-}+\bar{\nu}_{e}+\nu_{\mu} \\
\mu^{-}+(A, Z) \rightarrow e^{-}+(A, Z) .
\end{gathered}
$$

In the case of the free-muon decay, most of the muon energy is transferred into momentum for the resultant neutrinos. This leaves the electron with a momentum less than or equal to $55 \mathrm{MeV} / \mathrm{c}$. In the Mu2e conversion, all of the energy of the muon is supplied to the rest mass of the electron and its respective momentum. This means that the Mu2e conversion yields an electron with the specific momentum of $105 \mathrm{MeV} / \mathrm{c}$.

Mu2e is an Intensity Frontier experiment at Fermi National Accelerator Laboratory. The Intensity Frontier is one of the three broad characterizations of HEP experiments, which also includes the Cosmic Frontier and the Energy Frontier. The Cosmic Frontier deals with a larger picture of the universe, and attempts to discover the nature of dark matter and dark energy. The Energy Frontier, obviously enough, involves the highest energy HEP experiments. These experiments seek to recreate the first billionths of a second our universe experienced, in order to discover new particles and understand their interactions. Finally, experiments in the Intensity Frontier, like Mu2e, are concerned with a more thorough study of elementary particles, inspecting their quantum numbers and mass relationships. Instead of using extremely high energy like experiments in the Energy Frontier, the Intensity Frontier requires precision through a finely tuned apparatus and a large number of interactions.

Again, the goal of Mu2e is to observe an instance of CLFV via the conversion of an electron to a muon without any neutrinos. Though alternate theories predict this 
coherent conversion at higher rates than the SM, it’s still extremely unlikely to be observed. Therefore, in order to observe such an elusive process, a finely tuned design is essential. Specifics regarding the design will be discussed in detail in the third chapter. Mu2e hopes to begin taking data in 2021, nearing the end of its five year construction process. Before construction is finished, collaborators will continue to spend time focused on running various simulations focused on increasing the precision of the experiment. This includes simulating physical backgrounds which will help us understand future data and help calibrate equipment. Once construction is complete, we'll execute different simulations regarding the analysis and management of data.

\subsection{Radiative Pion Capture}

A false-positive signal is one of the more worrisome aspects of conducting a high precision experiment focused on observing an extremely rare process. Thus, many collaborators spend time focusing on potential sources of background which would cause a false-positive signal. Though there are many potential sources of background in Mu2e (cosmic rays, antiproton annihilation, etc.), this study will concentrate on one specific form of background: radiative pion capture via Aluminum nuclei.

In theory, the conversion electron Mu2e seeks to observe must have a specific momentum of $105 \mathrm{MeV} / \mathrm{c}$ (in lab frame). This value is reached by converting the rest mass of a muon into the rest mass of an electron with a specific associated momentum (105 MeV/c) supplied by the remaining energy. This means that any process yielding an electron with a measured $105 \mathrm{MeV} / \mathrm{c}$ momentum will fake the desired signal. Radiative 
pion capture (RPC) is a process that occurs in Mu2e, which is able to produce such a result. This process occurs when a pion is captured by nuclei of a material, which is an Aluminium target in the case of Mu2e. We see this happen more often in the form of an electron being captured by nuclei yielding a photon. However, in the case of the more massive pion, its yielded photon has more energy than that of a photon resulting from radiative electron capture.

Once a photon is generated from RPC, this photon is able to undergo the previously mentioned process known as pair-production. Pair production of a photon often results in an electron-antielectron pair, and the electron in this scenario is able to have a momentum of $105 \mathrm{MeV} / \mathrm{c}$. Since the possibility of RPC occurs near the detector, this is a major source of potential background. 


\section{CHAPTER 2}

\section{THEORY}

\subsection{Supersymmetry}

If the SM claims that the Mu2e process has an ultra-low probability, then what drives researchers to explore such a phenomenon? This section will cover the motivation behind Mu2e with a brief overview of the alternate theories. Supersymmetry (SUSY) is the family of alternate theories which provides Mu2e a basis for observing the coherent conversion of a muon to an electron.

\subsubsection{The Hierarchy Problem}

A fundamental flaw of the SM is its inability to adequately explain the gap between the gravitational force and the weak force. The "hierarchy problem" gains its name from the discrepancy between the gravitational force and the other three fundamental forces. To understand this gap, let's closely inspect the weak and gravitational forces. The mass of the $\mathrm{Z}$ and $\mathrm{W}(+,-)$ bosons, responsible for the weak force, have been measured at $\sim 100 \mathrm{GeV}$, which fixes the Higgs field energy value at 250 GeV. Initially, this seems acceptable, as 250 and $100 \mathrm{GeV}$ are close in value; however, the truth is rather counter-intuitive. 
The core concepts of Quantum Field Theory and Quantum Mechanics tell us that all properties of matter must be quantized. This concept gives us the smallest unit of time "Planck time”, the smallest possible unit of length "Planck length”, and the less mentioned "Planck mass". Of course, these names all pay homage to a key co-founder of Quantum Mechanics, Max Planck.

Turning our attention to the previously mentioned "Planck mass", one might ask "Is this defined as the smallest unit of mass?”. The befuddling answer to this question: no. Planck mass is defined as the smallest possible black hole able to exist, which would theoretically yield a Schwarzschild black hole with radius of Planck length. Therefore, if the Planck mass is related to gravity's standing within the SM, then it should be of a similar order of magnitude to the other three fundamental forces. Unfortunately, this definition yields a Planck mass of approximately $10^{\wedge} 18 \mathrm{GeV}$. If gravity is to be represented accurately within the SM, then the value of the Higgs field energy must either be the trivial value (zero), or the Planck mass energy. We find the Higgs field energy ( $250 \mathrm{GeV})$ is not anywhere near Planck mass energy or the trivial value of zero, leaving us faced with the hierarchy problem.

The value of the Higgs field energy is calculated in Quantum Field Theory using standard quantum corrections from the particles in the SM. The only stable solutions to these corrections are the trivial solution and Planck mass energy. Here, SUSY finds its way of entry. At one point it was realized that the quantum corrections between each partner within the SM (supposing members of fermions and bosons have a natural symmetry) should equal the exact Higgs field energy value of roughly $250 \mathrm{GeV}$. This is the solution to the hierarchy problem offered by SUSY, as well as the theory's 
foundation. An interesting facet of SUSY is that it is inherently a broken system;

otherwise it's not physically realistic. If the symmetry was ideal, then the supersymmetric partners would have identical masses.

\subsubsection{Minimal Supersymmetric Standard Model}

The most conservative form of SUSY is the Minimal Supersymmetric Standard Model (MSSM). From its name, one can surmise that MSSM is only a minor extension of the basic SM. There are two central features in MSSM which differ from the SM: (1) there is one family of supersymmetric particles, and (2) the symmetry is softly broken. This slight divergence from the SM means that testing the MSSM theory is quite difficult. For instance, one simple way of testing MSSM would be to measure particles proposed to be super partners. Unfortunately, no recent experiments have been able to do this, as this would require energy beyond what is currently available for experiments in the Energy Frontier.

As mentioned in the preceding chapter, alternate theories (specifically MSSM) state that the conversion of a muon to an electron occurs at a higher rate than the SM, which places that probability at $10^{\wedge}-52$. MSSM speculates that the muon is more intimately related to the electron than what the SM indicates. The SM already perceives the muon as the slightly more massive cousin of the electron, so it's not out of the question that the two may be more connected. Beyond Mu2e, there are a number of other experiments involving processes that, if observed, would point to physics beyond the SM, and would reinforce the MSSM theory. 


\subsection{Radiative Pion Capture: External and Internal Conversion}

The nuclear capture of a negatively charged pion by an Aluminium nucleus is the focus of this background study. We look to the quark content of each particle involved in this process: one down quark and two up quarks creating a proton in the Aluminum nucleus, and one down and anti-up quark of the negatively charged pion. When the pion is captured by the nucleus, its anti-up quark annihilates with the up quark of the proton, resulting in a photon. The photon resulting from this interaction is able to pair produce and create an electron-positron pair where the electron is able to have a conversion momentum of $105 \mathrm{MeV} / \mathrm{c}$.

The quantum mechanical process of RPC can result in two different types of processes to create a photon, which are external conversion and internal conversion. The external conversion process results in the emission of an on-shell, or "real”, gamma-ray photon from the nucleus. This gamma particle either goes on to pair produce in the stopping target (or in other material in the detector solenoid region), or it exits the detector region without ever pair-producing. The internal conversion process involves the emission of an off-shell, or "virtual", gamma-ray photon from the excited nucleus due to pion capture. In this case, the virtual photon must pair-produce into an electron-positron pair. We can see that the internal conversion photons are more likely to produce undesired background for Mu2e; however, the probability of producing a real photon is far greater than that of a virtual photon. The result is that internal and external 
conversions contribute an equal probability of generating background. Separate simulations are carried out for external and internal processes. 


\section{CHAPTER 3}

\section{THE MU2E COMPLEX}

\subsection{Overview of Mu2e}

The basis for the muon to electron conversion hinges on the conservation of energy, as the rest mass of the muon is converted into the rest mass of an electron plus a specific value of momentum ( 105 MeV/c) for said electron. Excess energy from the mother (muon) is converted into momentum for the daughter (electron) out of necessity. Momentum has a vital role as it's an observable that we're readily able to measure, as

well as a value that definitively indicates the coherent muon to electron conversion. For this signal to occur, we must first find a method for the muon, specifically a low momentum muon, to be captured atomically. Collaborators at Mu2e have designed a stopping target (ST) made of Aluminium, which is monitored in the Detector Solenoid (DS).

In order to generate these muons, Mu2e uses protons from FNAL’s Main Injector to interact with our Production Target (PT) composed of Tungsten. Interestingly, the overwhelming majority of what's produced by the PT is pions, though there is a presence of muons and kaons (Mu2e Collaboration, 2015). Most of these pions will decay into a muon along with an antimuon neutrino, which are guided by solenoids that lead to the 
ST. These solenoids create a gradated magnetic field decreasing over distance, which effectively combs out muons of an undesired momentum. A portion of these muons are captured by the Aluminium target, and hopefully one or more undergoes a coherent conversion to an electron.

\subsection{Radiative Pion Capture}

As indicated earlier, not all produced pions decay into muons, and it's these pions at risk of undergoing RPC. Specifically, these pions can be captured by the Aluminium nuclei in our ST, and decay into a high energy photon. This high energy photon is able to undergo the process of pair production, spontaneously converting into an electronantielectron pair. The resulting electron is able to have a momentum of $\sim 105 \mathrm{MeV} / \mathrm{c}$, which would fake our desired conversion signal. Therefore, the RPC process is considered a serious source of potential of background to Mu2e.

\subsection{From Proton to Stopped Muon}

The upcoming subsections will detail select components of the Mu2e apparatus including a quick overview of Fermilab’s accelerator complex as it relates to Mu2e. Figure 3.1 shows the Fermilab complex with Mu2e highlighted, as well as the general sections of their beamline. Note that the proton beamline is located underground, while the Mu2e apparatus is located in a building. Figures and information in this section are 
provided by the Mu2e Technical Design Report and the Mu2e Collaboration 2016.

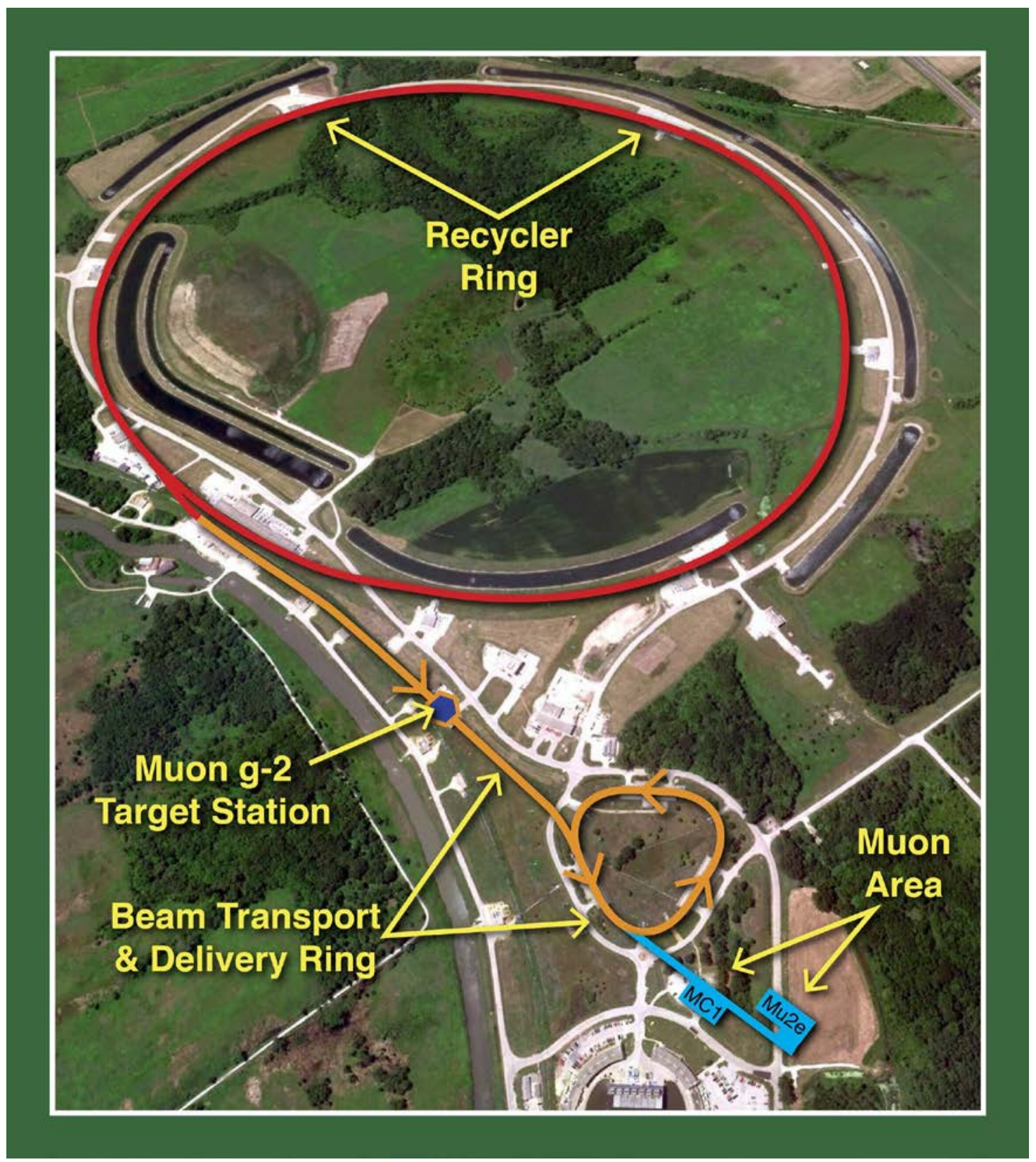

Figure 3.1: Overhead picture of Fermi National Accelerator Laboratory Proton Beamline

\subsubsection{Proton Beamline}


Mu2e uses the $8 \mathrm{GeV}$ proton beam at Fermilab, a relatively low energy when compared to other HEP experiments. For instance, the Large Hadron Collider in Geneva has a beam of $\sim 13 \mathrm{TeV}$, over three orders of magnitude above the Main Injector at Fermilab. This appears consistent as we discussed before that Mu2e falls under the Intensity Frontier, which isn't concerned with exceedingly high energy setups, but instead focused on precision. This low energy proton beam will yield low energy pions and muons, which optimizes the number of stopped muons, and increases the probability of observing a conversion electron.

Our proton beam is composed of separate pulses by design, which effectively prevents potential background. Pulsing the beam creates a Gaussian distribution with respect to time, providing separation between the peak of the pulse and the peak of potential background. In Figure 3.2 we find an optimal time window for the detector to collect data, excluding any data generated during the "prompt flash”. We'll revisit the prompt flash and provide data that yields this result in the following chapters.

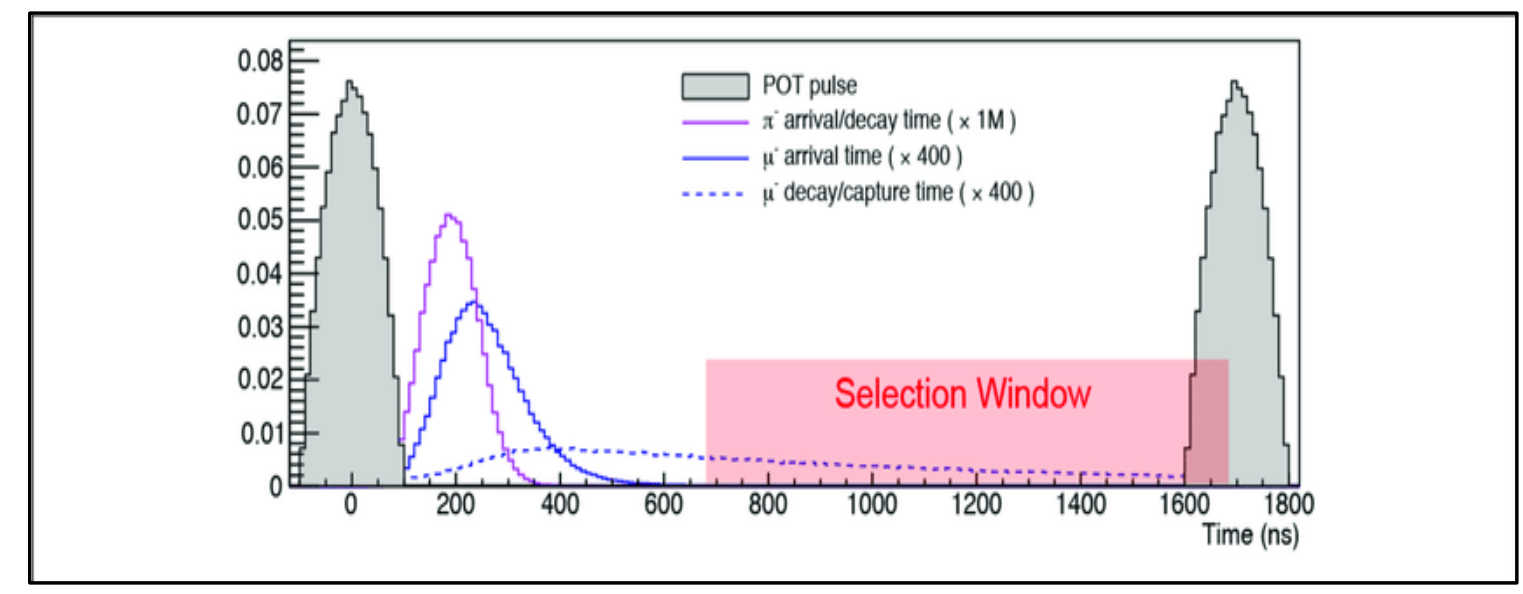

Figure 3.2: The proton pulse used in the Mu2e experiment. Also shown are the capture and decay time ranges for both muons and pions. 
The proton beam starts its journey at the FNAL Ion Source, starting out as hydrogen ions (H-). After being stripped of its electrons, the proton beam is given its initial boost from the Linac, Fermilab’s linear accelerator. Immediately following this, the beam travels to the Booster Ring (BR), where the beam receives a periodic kick, increasing the beam's kinetic energy until it reaches $8 \mathrm{GeV}$. Once the beam leaves the Booster Ring, it's fed into the Recycler as two batches where each batch is then divided into four distinct bunches via radio-frequency sequencing. These bunches are directed below the Recycler to the Main Injector, which are then transferred to the Delivery Ring (DR). As one might expect, the DR delivers the proton bunches to the PT, where each bunch will contain approximately 3 x 10^7 protons.

\subsubsection{Production Solenoid}

The Mu2e experiment is covered by three different solenoids (as seen in Figure 3.3) which act as a guide for low momentum muons to reach the ST, while simultaneously reducing other particles sent to the ST. The first of the three solenoid system is the Production Solenoid (PS), which surrounds the PT. Our PS has a magnetic field which ranges from $4.6 \mathrm{~T}$ to $2.5 \mathrm{~T}$, and this gradient decreases as the solenoid extends downstream. This gradient effectively filters out unwanted, positively charged particles, as Mu2e seeks to generate a beam of negatively charged muons.

Of the numerous particles generated by the interaction between PT and the proton beam, Mu2e intends to maximize the creation of negatively charged pions. These pions are then led towards the TS, where the bulk of the pions will decay into negatively 
charged muons. The geometry of both the PT and the initial area of the PS is designed to minimize the reabsorption of negatively charged pions, assisting in the optimization of pions reaching the TS. For reference, the lifetime of a muon is statistically much longer than that of a pion. Therefore, it's logical to design a beam consisting of both pions and muons, despite the fact that these pions are at risk of generating background. Lastly, when a pion naturally decays into a muon it is accompanied by an antimuon flavored neutrino.

When discussing the PS, the extinction monitor must be mentioned. Located near the PS area, the extinction monitor measures the number of protons which reach the PT at a time outside the pulse window. These stragglers are aptly referred to as out-of-time (OOT) protons, and are a potential source of background radiation via RPC. Protons within the bunch are referred to as protons on target (POT). The extinction monitor essentially checks whether or not the proportion of OOT protons to POT is at an appropriate level. The required ratio of OOT protons to POT, referred to as the extinction factor, is to be below $10^{\wedge}-10$. The current predicted extinction factor via simulations is roughly $10^{\wedge}-12$, two orders of magnitude below the required value.

\subsubsection{Transport Solenoid and Collimator}

The previously discussed TS follows the PS, and its primary function is to filter out high momenta muons, and corral muons of a desired charge and momenta to the ST. The TS is strategically designed in an S-shape (Figure 3.3), and like the PS, this solenoid has a gradated magnetic field ranging from $2.5 \mathrm{~T}$ to $2.0 \mathrm{~T}$ as it extends downstream. Both 
of these design characteristics aide in two ways to the primary function of the PS. Particles with momenta above a deemed threshold will eject tangentially during the first curved section of the solenoid eventually crashing into walls as the magnetic field fails to usher it beyond the bend. On the other end of the spectrum, particles with momenta below a certain threshold will be deflected too much by the magnetic field during the curve.

Within the TS area are collimators, which filter the beam based on the charge and momentum of a particle. The primary collimator, located in the straight section of the TS, seeks to block positively charged particles and accept negatively charged particles. During this bend, charged particles experience an induced electric field due to the toroidal shape of the curve. This induced electric field separates positive and negative charges allows the central collimator to permit only negatively charged particles via a simple window. This means the central collimator plays a crucial role in optimizing the number of negatively charged particles within the beam.

\subsubsection{Detector Solenoid and Stopping Target}

The above-mentioned DS, like the PS, is a straight solenoid containing a gradated magnetic field ranging from $2.0 \mathrm{~T}$ to $1.0 \mathrm{~T}$ as it moves downstream. The ST is located at the beginning of the DS, near the TS. The gradation of the DS occurs quickly as the field reaches $1.0 \mathrm{~T}$ at the site of the ST, and this $1.0 \mathrm{~T}$ field remains throughout the remaining DS area. Within this uniform 1.0 T field resides the detector system. The reason for this uniformity is to allow the signal electrons to travel in a well-defined, helical path. 
The ST is comprised of 37 rings of Aluminium foil with uniform radii with an inner circle removed from each foil. Each foil is to be $75 \mathrm{~mm}$ in radius, $0.1 \mathrm{~mm}$ thick, and spaced approximately $22 \mathrm{~mm}$ apart. The face of this cylindrical structure will be situated perpendicularly to the beam line. The ST geometry has been designed to optimize the stopped muon yield and minimize the reabsorption of potential signal electrons.

\subsection{The Detector Complex}

The following section will focus on the components of the detector system, as well as honing in on details regarding the muon to electron conversion process.

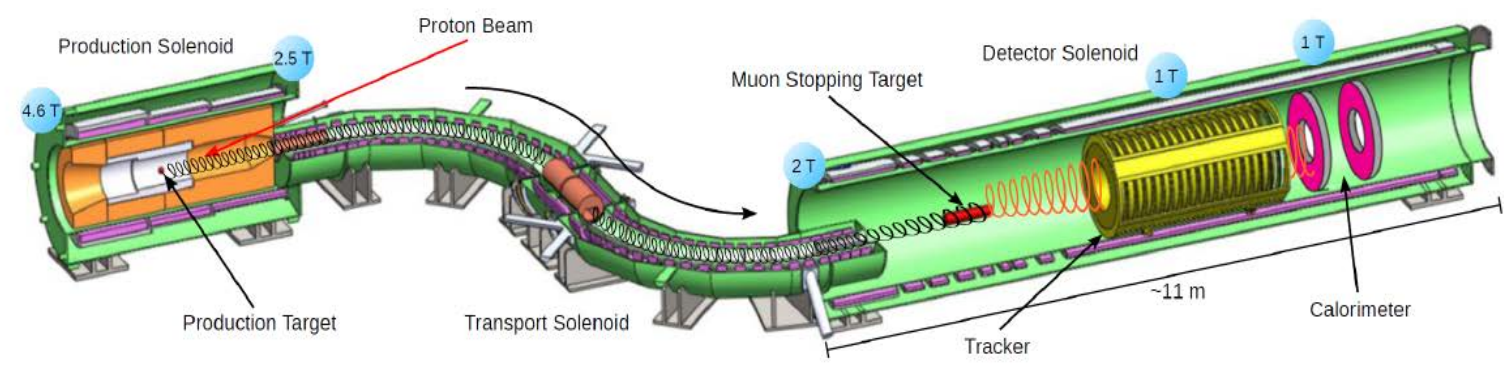

Figure 3.3: The Mu2e experiment apparatus

\subsubsection{Stopping Target Monitor}

The Stopping Target Monitor (STM) is self-explanatory as its singular function is to count the number of stopped muons in the ST. A conventional method of acquiring this data would be to measure the photon spectrum produced by the atomic capture of muons; 
however, the background coming from the prompt flash would interfere with this measurement. Instead, Mu2e focuses on the photon spectrum produced by nuclear muon capture, which is slightly delayed in comparison to the atomic muon capture. This ever so slight delay provides the STM enough time to take the measurement without backgrounds present. With this information we can extrapolate the total number of muons stopped. An important note here is that the Mu2e process will occur atomically, and will not happen when the muon undergoes nuclear capture.

\subsubsection{Tracker}

The first component of the detector system is the tracker. A majority of HEP experiments rely on track reconstruction, which is logical as trackers detail the position of individual particles with respect to time. The specific trajectory of a particle allows researchers to determine information about the particle, such as transverse momentum calculated via the Lorentz force. Most tracker systems use the ionization of a charged particle as it passes through a known medium in order to measure the position. The Mu2e tracker (detailed in Figures 3.4 and 3.5) uses 21,000 straw drift tubes of 5 mm thickness Mylar® tubes, and 25 um thick sense wire situated concentrically within each other. These tubes will have spacing within them filled with Argon gas, allowing the ions to drift to the sense wire and measure position of charged particles (Ciampa, 2018). The geometry of these tubes is constructed such that conversion electron's specific trajectory will be uniquely tracked relative to that of non-conversion particles, shown in Figure 3.5. 


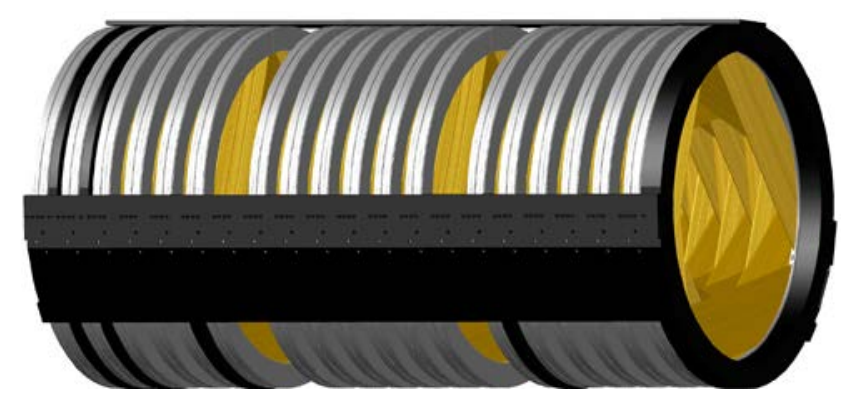

Figure 3.4: The tracker system used in the Mu2e detector system

One critical aspect of the tracker design is its radius. As mentioned in the previous paragraph, the geometry of the tracker is strategically engineered to measure conversion electrons in a unique fashion. The signal of a conversion electron will have a specific momentum (105 MeV/c), which will be represented in the radius of the helical trajectory measured by the tracker. This is due to the Lorentz force experienced by the electron in a static magnetic field, with a specific value of velocity. It is wise to note that this value of velocity corresponds to the transverse momentum of the conversion electron, not the magnitude of momentum. Despite this fact, the vast majority of potential background will have a low momentum. Let's suppose that the particle has its momentum completely in the transverse direction; even with this weighting, the particle wouldn't produce a similar result to the conversion electron in the tracker. Unfortunately, this constraint of the tracker results in the possibility of missing a small number of potential signal tracks; however, this is offset by the background rejection achieved.

\subsubsection{Electromagnetic Calorimeter}




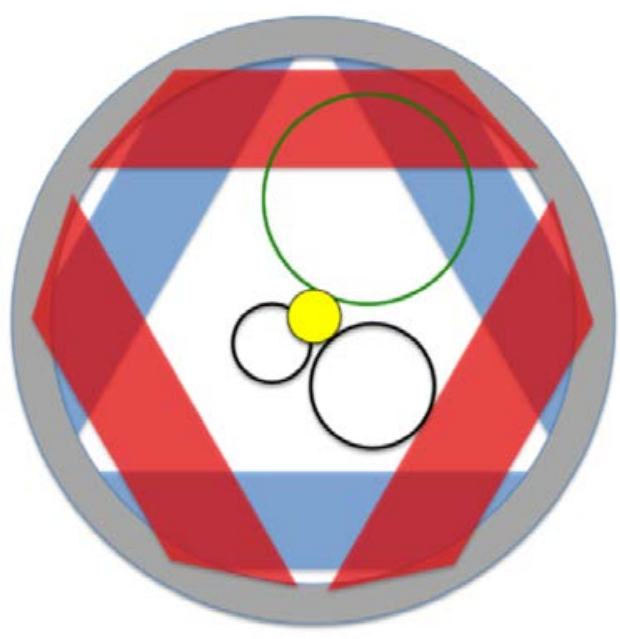

Figure 3.5: Different radii of electrons of different momentum. The green circle in the top right is the radius of the trajectory for an electron with momentum of $105 \mathrm{MeV} / \mathrm{c}$. The bottom right circle is the radius of an electron produced in decay in orbit with a momentum of roughly $50 \mathrm{Mev} / \mathrm{c}$. The bottom right circle is the radius of trajectory for an electron with a momentum of less than 50 $\mathrm{MeV} / \mathrm{c}$. The yellow circle in the middle is the stopping target.

The principal purpose of the calorimeter is to identify particles and act as a fast signal for triggering. Data from the tracker will be used in Mu2e Online software to create track reconstruction based on the "hits", representing the position of charged particles, in the tracker. In reality, the tracker experiences a vast number of hits within minute separations of time, which have the potential to create a false track reconstruction. Since many tracks will have energies close to the desired signal energy, the possibility of a mis-reconstructed track appearing consistent with a muon to electron conversion is likely. In these instances, the calorimeter will be able to confirm or deny this signal based on its own independent measurement of both energy and momentum. 


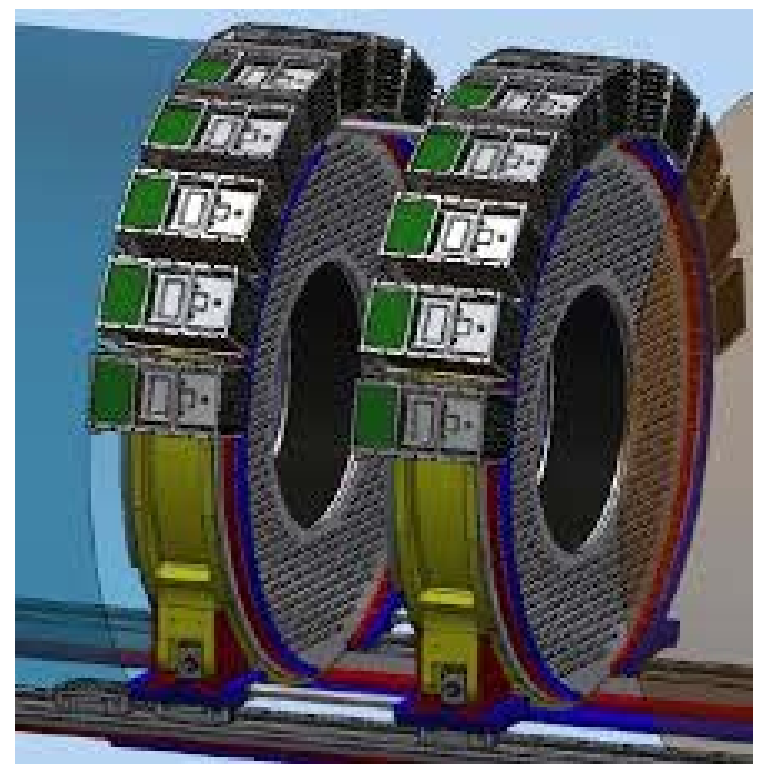

Figure 3.6: The Mu2e Calorimeter

The calorimeter used in Mu2e is designed to interact with charged particles, stop them, and completely absorb their energy. Our calorimeter is composed of Silicon Photomultipliers (SiPMs) and Cesium Iodide (CsI) crystals. When the CsI crystal interacts with charged particles, photons are emitted and consequently collected by the SiPMs. Directing our attention to Figure 3.6, we see that the calorimeter is constructed out of two ring-shaped disks. Each ring is composed of CsI disks with an array of 640 CsI crystals. Each side of the disk is $3.4 \mathrm{~cm}$ per side, and $20 \mathrm{~cm}$ deep. The SiPMs are attached downstream (not shown in Figure 3.6). Charged particles interact with the calorimeter yielding photo-showers in the CsI crystals. The timing resolution of the SiPMs will then be able to identify the stopped particle (Atanov, et al. 2017).

\subsubsection{Cosmic Ray Veto}


The Cosmic Ray Veto (CRV) plays a critical role in Mu2e’s detector system as its job is to obstruct cosmic rays from creating unwanted background. To review, cosmic rays are particles generated by various initial interactions in the universe, which take place outside of Earth's atmosphere. These particles are born out of a variety of interactions, meaning that their associated energies are wildly variable. Most particles able to travel to the Mu2e detector without decaying are muons. Since these muons are charged particles and can possess energies near the signal energy, cosmic rays are considered a serious background potential, which would effectively trick both the tracker and the calorimeter.

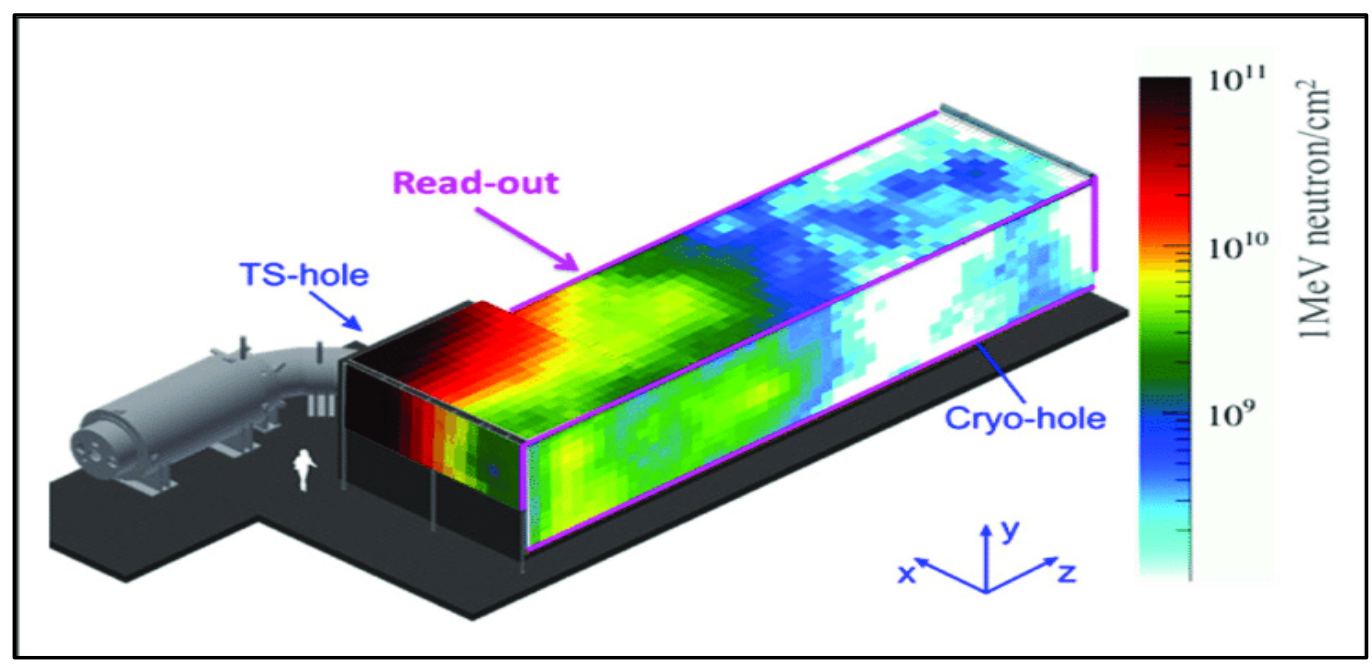

Figure 3.7: The Cosmic Ray Veto in the Mu2e experiment

As shown above in Figure 3.7, Mu2e's first line of defense for cosmic rays is its built in shielding on every vulnerable side of the DS. For cosmic rays with enough energy to penetrate this shield, Mu2e can reject this potential background via particle identification provided by the tracker and calorimeter. Moreover, there is the ever so 
slight chance that a particle could pass through the CRV, interact with the ST, and emanate an electron with a momentum of $105 \mathrm{MeV} / \mathrm{c}$. This type of process would be undetected as track reconstruction would show that the conversion electron came from the ST. To combat a particle of this nature, the aforementioned shield is composed of Silicon photo-multipliers (SiPMs) in such a way that they can be used to detect incoming cosmic rays. These SiPMs will measure the energy of incoming cosmic rays, and if this observed energy is within a certain range, the CRV will trigger a "veto" for the detector. 


\section{CHAPTER 4}

\section{SOFTWARE \& SIMULATIONS}

\subsection{Software - Frameworks \& Packages}

To achieve the level of precision required in modern HEP experiments, simulations must be used in every conceivable aspect. This ranges from simulating the effect of geometry, to the simulation of potential background. The RPC study would not be possible without these methods of simulation, which have been advanced over the decades by previous HEP experiments. Without packages like Geant4 (G4) and Monte Carlo (MC), HEP experiments would be extremely limited. In the case of Mu2e, critical software includes Mu2e Offline, G4Beamline, and MARS. G4beamline is a software toolkit which enables researchers to build detector components and the buildings which house detectors. MARS is used to simulate radiation from different sources, allowing researchers to determine how safe the environment is for both humans and components of the detector.

Mu2e Offline is the primary software package used to simulate the process of RPC and understand its effect on Mu2e. Fermilab’s art software framework is the foundation of Offline, and will be detailed in the upcoming subsections. 


\subsubsection{Fermilab's art Framework}

The art event processing framework is Fermilab’s unique, but highly standardized software which was created to have a wide range of functionality. As an event processing framework, the overall reach of art is within the detection process. Several critical aspects of Intensity Frontier experiments are addressed by the art framework, including: calibration, high level software filters, reconstruction, simulation, analysis, and online data monitoring (Green et. al, 2012). At the current moment, Mu2e Offline applies art to both simulations and analysis. From the start of its development, art was designed to be utilized by numerous different experiments. Past experiments would work within their own framework and have that coupled closely to their unique code. Unsurprisingly this led to decreased efficiency as similar tasks were repeated across multiple experiments, effectively duplicating efforts.

As a framework based on the programming language $\mathrm{C}++$, art has unique classes referred to as modules. These modules allow researchers working on Mu2e to use this framework. Within each art module exists a number of classes used in the code of each experiment. Modules are then configured in text files in Fermilab Hierarchical Configuration Language, ending in .fcl (pronounced “fickle”). Below in Figure 4.1, we see an example of what an elementary .fcl file looks like. In order to run this file, we can use a simple command such as, “art -c example.fcl”. In this instance, the .fcl file informs art which modules need to be used, and the parameters associated with each module.

The hierarchy of information from within the art framework, from top to bottom, 
is as follows: jobs, runs, subruns, and events. Each module in a .fcl file must have code that is called at least once for each event.

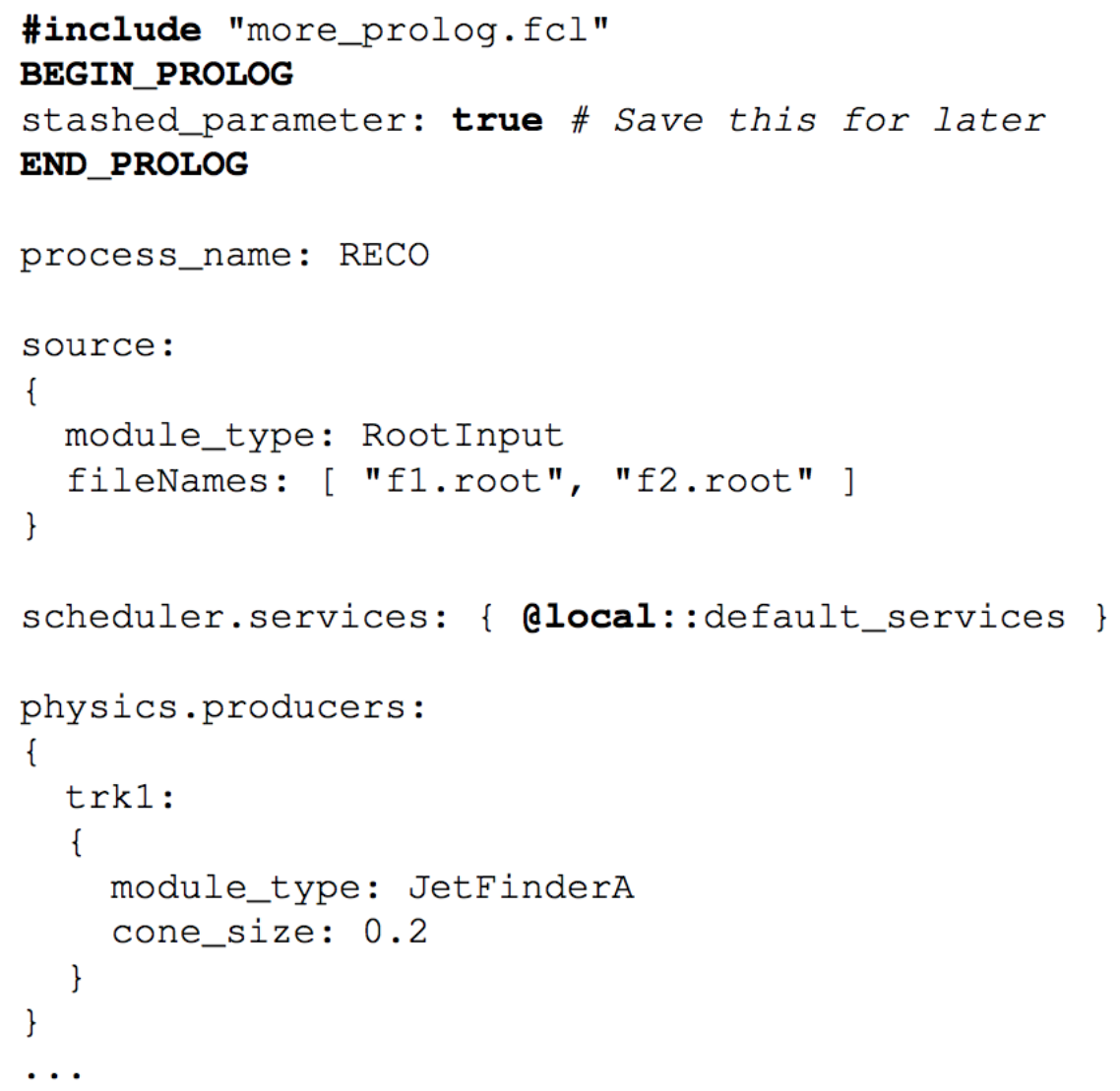

Figure 4.1: An example of a simple .fcl file (Green et. al., 2012)

Whether this code is called at the beginning or end of a subrun, run, or even job, is variable. Each module within the art framework must be one of five types, depending on how they interact with events. There are five unique modules within the art framework to compose .fcl files: 
- analyzer module: able to observe information found in the event, but cannot alter information.

- producer module: able to both observe information in an event and add information to an event.

- filter module: same abilities as producer module, but also able to instruct art to skip processing of certain modules for the event.

- source module: reads events individually from a specific source, and art demands every job has one source module. An example of a source is a disk file.

- output module: writes selected data products from memory to an output destination

Unlike modules in art which execute tasks, data products are C++ classes that collect data from either modules in a simulation or from the data-acquisition process. Experiments have self-defined data products, which makes sense as these classes are passive. Another important type of special $\mathrm{C}++$ class used in the art framework is services. Services in art is a configurable utility class registered in the framework, which can be accessed from modules through a ServiceHandle. Services control the creation and destruction of service objects, as well as verifying that the number of service objects are available. Modules and services fall under the category of plug-ins, which means that these classes exist within libraries. These classes are made available to programs once configuration files have called them. The flow of an art job is handled by a state machine, the EventProcessor. This manages the setup of the primary application and the calling of services, as well as the stages of each module. To end the discussion of art, we 
must talk about "external products” within the framework. External products are software packages separate from both user code and art, such as the FHiCL package which allows users to execute art modules. Another essential external product is ROOT, a software package used extensively in HEP experiments allowing users to manage vast datasets. In the following sections, we will examine histograms created using the ROOT software, which relate to the RPC process.

\subsubsection{Mu2e Offline}

As we had discussed earlier, Mu2e’s proprietary software package is called Offline, which is responsible for most of the work done by researchers. Offline, as one might suspect, has been developed and maintained by Mu2e, and is not used in other experiments at Fermilab. This section will detail the types of directories and subdirectories within Offline most frequently used by researchers in Mu2e.

Mu2e Offline has over 70 code containing directories, which employ a similar structure of having an src subdirectory containing specific source files (.cc). Other subdirectories such as inc and fcl may exist within these directories, which would contain .hh and .fcl files respectively. We can group these directories into five distinct types:

- Utility/Service directories: directories containing code with various support functionalities.

- Data product directories: specialized directories that contain various data products, which include SimParticle and StepPointMC. SimParticle will represent actual particles, whereas StepPointMC will represent individual points on a track. 
- Tracking directories: directories with code relating to simulating the tracker as well as the tracker's performance during data collection.

- Reconstruction directories: directories containing code which uses tracker hits, calorimeter clusters, and tracker-calorimeter matching in order to simulate track reconstruction.

- ExperimentComponentGeom directories: directories containing code relating specifically to the geometry of components comprising the experiment's apparatus.

One directory essential to the RPC study (along with other background studies) is the JobConfig directory. Within this directory are .fcl files used to monitor backgrounds as well as text files containing the Mu2e geometry code. All of the .fcl files used in the RPC study can be located in the JobConfig directory.

\subsection{Simulations}

This section will explain the various stages of simulations specifically used in the RPC background study. This section will identify which .fcl files are used during each stage of simulation, as well as the various quantities involved. The simulation process for the RPC study is broken into four distinct stages: g4s1-g4s4. These stages start at simulated protons-on target (POT’s) all the way to simulated RPCs generating a "fake" signal. 


\subsubsection{From POT to Stopped Pion}

As mentioned in the previous chapter, pions generated from POT’s will ideally decay into muons at some point within the TS, and a number of these muons will interact with the ST. Pions that do not decay into muons (or other particles) are the source of RPC background. In order to model the effect of RPC on Mu2e, we begin by simulating POT's interaction with the PT. We then allow the resulting particles to travel through the system of solenoids via Geant4, and pions are not allowed to decay in these simulations. The proper time, a measure of time from the reference frame of the pion, is measured and stored. This information will enable us to weight stopped pion samples based upon their probability of survival. These pions eventually reach the ST and yield values of proper time, time, and position of the stopped pions. These resultant values are then used as input for following stages of simulation.

The previously described process is separated into three stages of simulation: g4s1, g4s2, and g4s3. G4 in these names denotes the fact that Geant4 is used in each stage, and the number following " $s$ " indicates the particular stage of the simulation. Thus, the first stage of this simulation is labeled g4s1, and simulates $10^{\wedge} 10$ POT’s with the same value of time (using the file pions_g4s1.fcl). Although this does not realistically reflect the nature of our POT pulse, this decision will be explained in the analysis. The resulting particles from the interaction of POT's with the PT are then simulated down to the central collimator in the TS. These particles are then killed and their SimParticle/StepPointMC information is recorded. Certain non-pion particles were cut from the simulation in order optimize efficiency, while leaving our RPC study 
unaffected. All neutrinos, as well as particles leaving the solenoid system, are cut as they have no ability to influence a pion's journey to the ST. Photons, electrons, and positrons with a kinetic energy lower than $100 \mathrm{MeV}$ are also cut from the simulation as they pose a negligible risk of affecting pions.

The following stage, g4s2, configured with the file pions_g4s2.fcl uses the result from g4s1 and generates pions exactly where they were killed. These pions have the same respective kinematic information as they did in g4s1 upon termination. Just as in stage 1 , these pions are allowed to move downstream to the DS where they are, again, simultaneously killed and recorded.

The final stage of this section, g4s3, is configured using pions_g4s3.fcl, and is responsible for getting the surviving pions to the ST. This third stage is also tasked with simulating the interaction between pions and the ST, yielding a value of pions stopped in the ST. This data, represented by a .fcl file, is then used in ROOT to build an ntuple. An ntuple organizes large sets of data in a straightforward fashion. Ntuples break down position measurements into their respective dimensions $(\mathrm{x}, \mathrm{y}, \mathrm{z}, \mathrm{t})$, in a way where we're easily able to isolate relevant dimensions. Specifically, in the study of RPC, we focus on the z-position of stopped pions.

In Figure 4.2 we see the position of stopped pions in the $\mathrm{z}$ dimension (downstream). An inverse relationship between distance and stopped pions exists as fewer pions are stopped as they move through the stopping target. Redirecting our attention to Figure 4.3 and 4.4, we see a histogram of stopped pions in the $\mathrm{x}$ and $\mathrm{y}$ dimension. While these individual plots are not terribly intuitive, if we plot pion stops in the $x-y$ plane the resulting graph should roughly resemble the stopping target. The 
plotting of pion stops in the $x-y$ plane can be seen below in Figure 4.5. This plot resembles a disk with a hole in its center, which is the exact geometry of our stopping target.

\subsubsection{Stopped Pion to Pair Production}

The fourth and final stage in this simulation is g4s4, which uses the output from g4s3 to simulate stopped pions undergoing RPC. Specifically, results from g4s3 give us the position of stopped pions in the ST. As previously stated, these pions are able to undergo two different types of conversion. The external conversion of a pion results in a real photon (which may or may not pair produce), while internal conversion results in a virtual photon which must pair produce into an electron-positron pair. Internal conversion photons will pair-produce immediately allowing us to conveniently simulate these resultant electrons with the same initial value of time.

One constraint placed on these photons is that they must have energy between 100 and $140 \mathrm{MeV}$. The energy spectrum of these photons (both external and internal) is assumed to follow the Bistirlich distribution. Using the previously mentioned cuts and distribution of energy, we're able to simulate the conversion process as well as the path of each daughter electron through the tracker and calorimeter. 


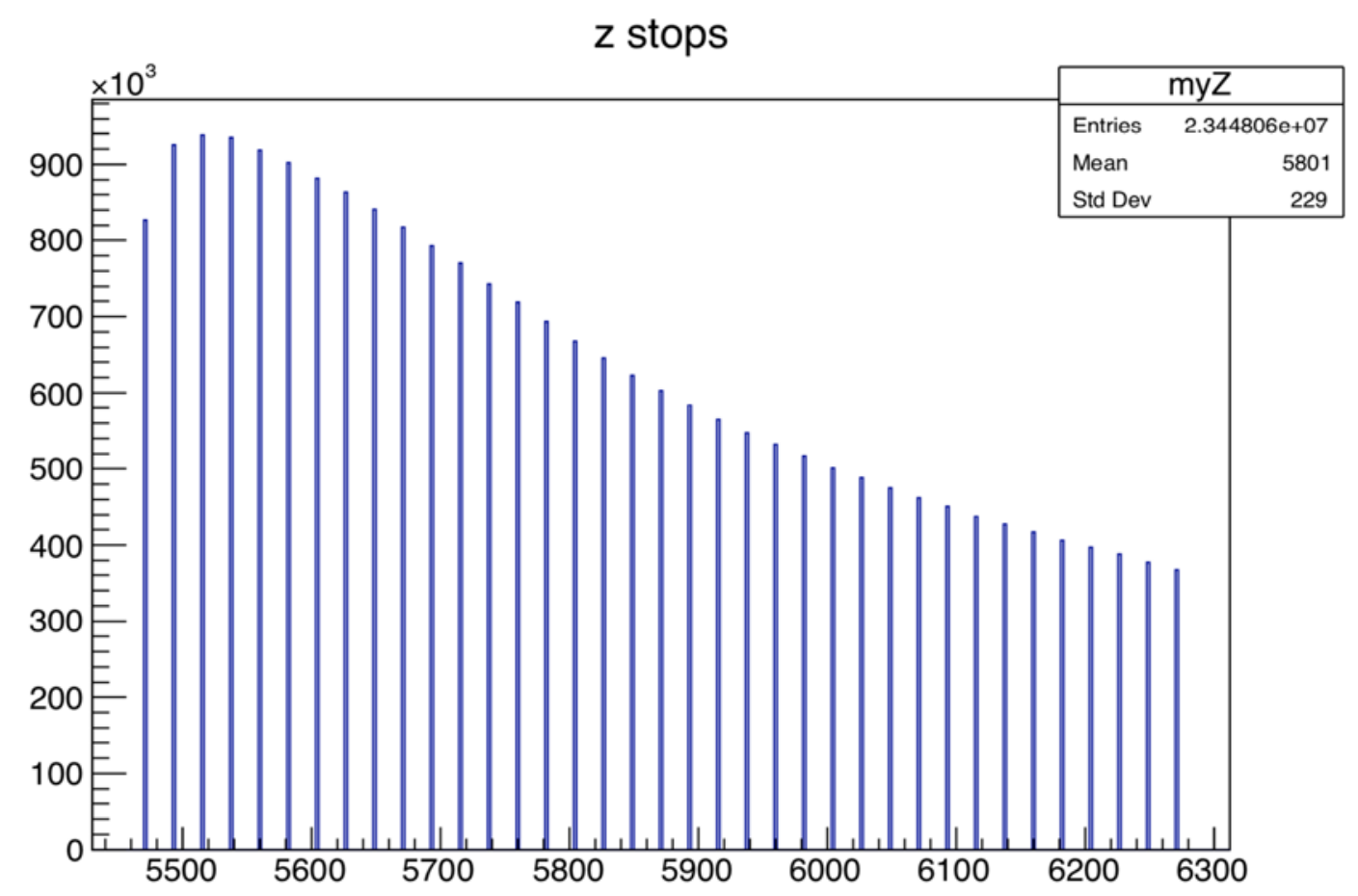

Figure 4.2: Histograms of stopped pions in the z-dimension of our stopping target

\subsubsection{Tracker Hits, Reconstruction, and Analysis}

Once a photon has pair-produced, be it internal or external, g4s4 simulates the path of the subsequent electron from ST to the detector system via hits recorded in g4s3. Observables such as momentum and energy are collected by the tracker and calorimeter, and these values are the final measurements of RPC simulations. We use these results, along with reconstruction packages within Offline, to create track reconstruction. Before we reconstruct the path of electrons, we must apply certain cuts in order to optimize the output of these simulations. In the following paragraph, we'll detail these various cuts.

Several cuts and restrictions are applied to tracker and calorimeter hits in order to create an accurate reconstruction of potential background. First, cuts are applied to hits that are unable to generate a consistent track. Second, if tracks have a track quality falling 
outside of a certain range ( 0.4 to 1.3 ) then they are cut. Next, the polar angle distribution of conversion electrons must be between 45 degrees and 60 degrees. Polar angles outside of this range include high energy electrons which will cause undesirable background. The subsequent cut restricts the time associated with the first hit in a track under $1695 \mathrm{~ns}$.

This restriction helps establish a well-defined observation period for the detector system. As discussed in the previous chapter, our detector system collects data during specific windows based on our POT pulse. The goal is to collect data during a period where RPC background is minimized, and the desired signal is maximized. 


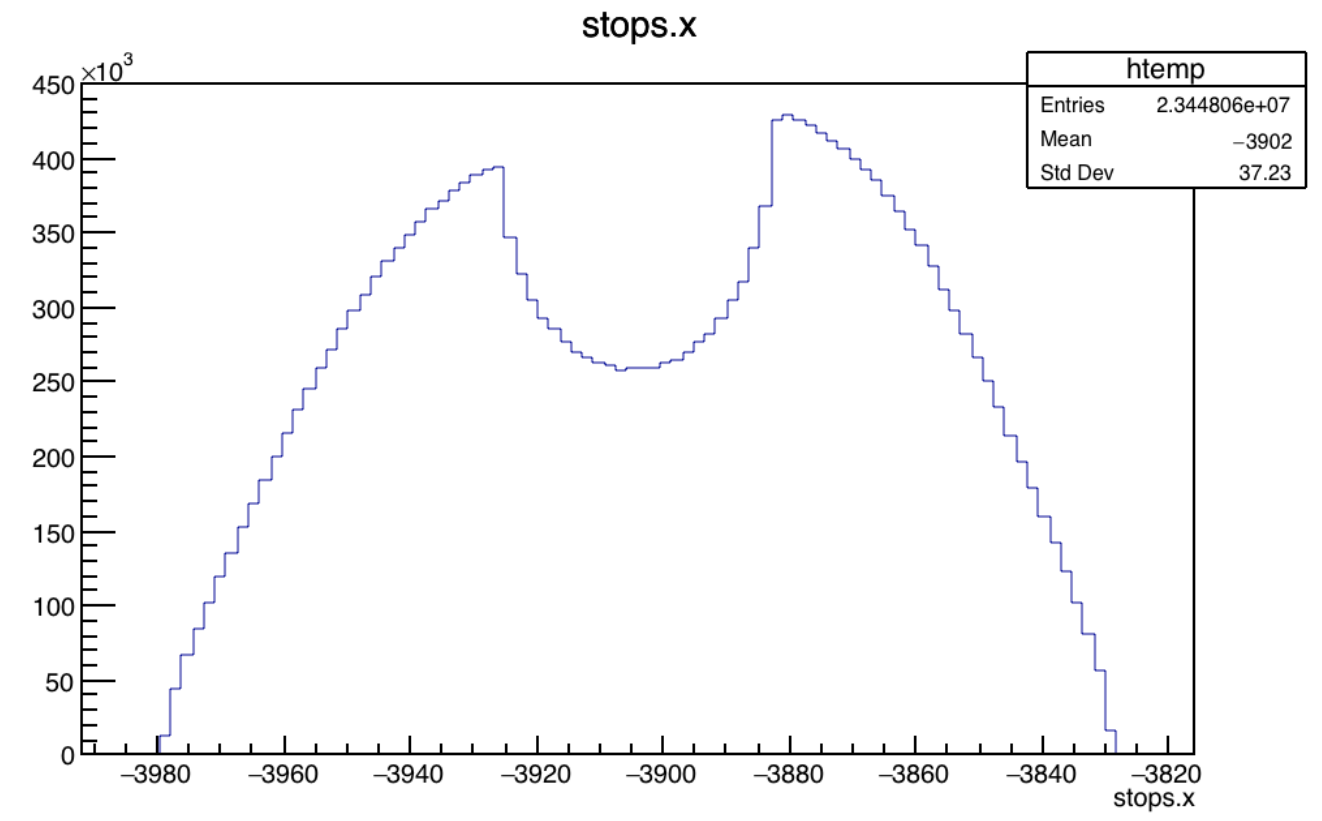

Figure 4.3: Histogram of stopped pions in the x-dimension of our stopping target 


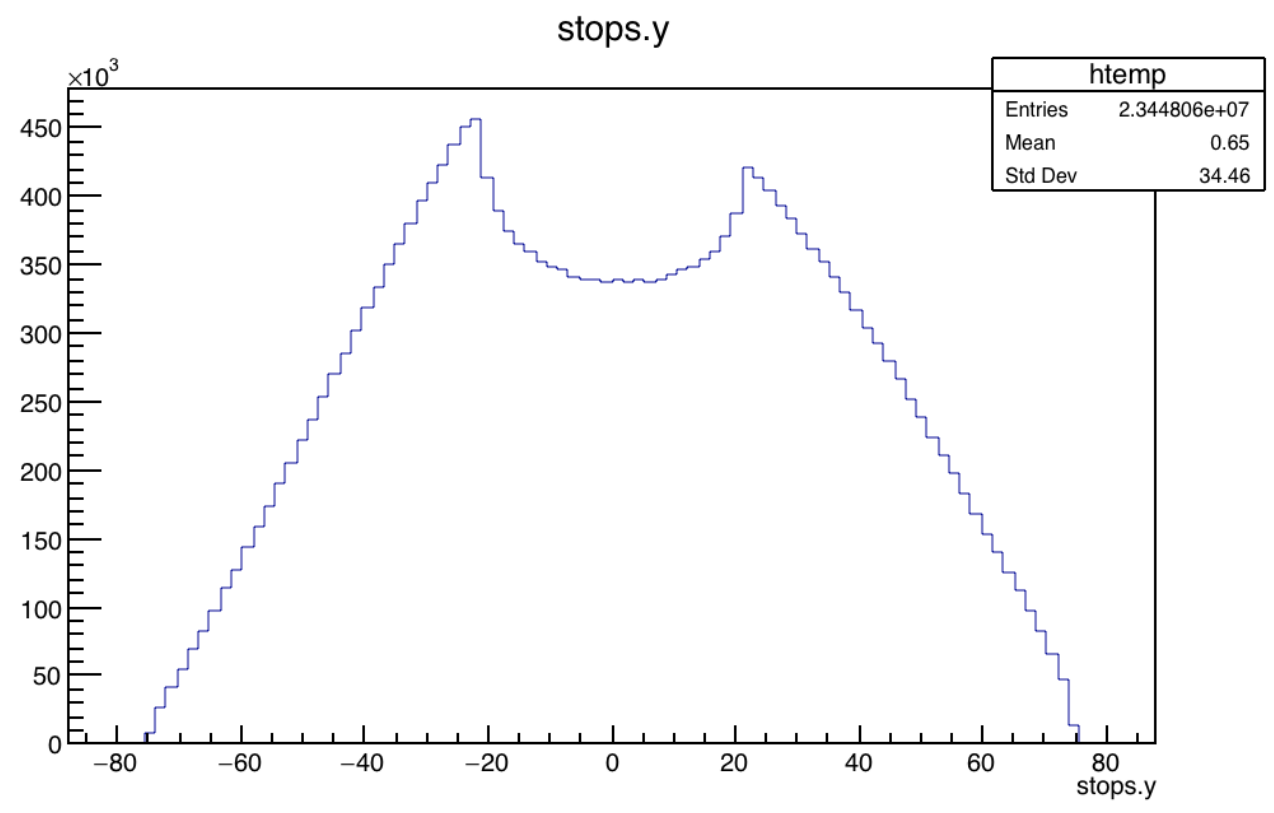

Figure 4.4: Histogram of stopped pions in the y-dimension of our stopping target 


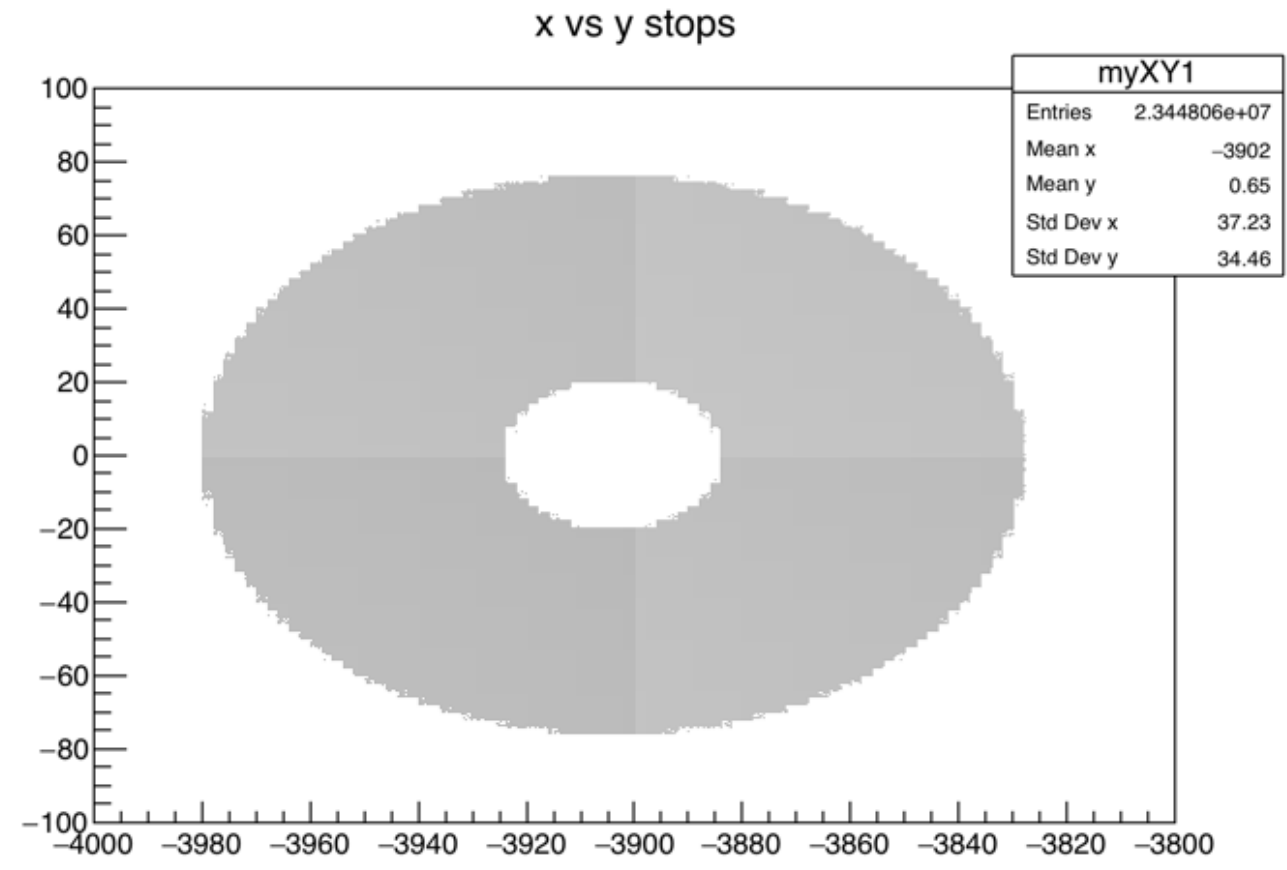

Figure 4.5: Histograms of stopped pions in the xy-plane of our stopping target 


\section{CHAPTER 5}

FINAL RESULTS

\subsection{Pure External \& Internal Conversion Results}

The following chapter will detail the results from stage four of the simulation. The tables below are the output from grid jobs using digital analysis reconstruction (DRA) files.

\subsubsection{Pure External \& Internal Conversion, In time}

First, we begin by looking at simulations involving pions resulting from in time protons. Data from varying cuts of time are recorded between 500 ns and 750 ns, when an RPC signal is most likely to occur during moments of the prompt flash (mentioned in Chapter 3). As a reminder, our prompt flash is the time period within the pulse when background is most likely.

External simulations had an input of 995 billion photons, and internal simulations began with an input of 994 billion photons. From these results, the time range most likely to experience an RPC signal is 500 ns, which has a value of 143 conversions for externally produced photons throughout the lifetime of the experiment. For internally 
produced photons, $500 \mathrm{~ns}$ to $550 \mathrm{~ns}$ is also the time range in which conversion electrons occur most. This value is slightly lower than external photons at 113 conversions over the lifetime of the experiment. However, as we approach the end of the prompt flash, it's apparent that the number of conversions drops off significantly. For example, for external conversions we have .00052118 conversions. This low value gives evidence that RPC background will not affect what is detected during data collection.

\section{TABLE 5.1}

Background due to In-Time Protons for both External and Internal Conversion Processes

\begin{tabular}{|c|c|}
\hline Time (ns) & Signals \\
\hline External & \\
\hline 500 & 143.728 \\
\hline 550 & 12.9345 \\
\hline 600 & 0.41563 \\
\hline 650 & 0.083771 \\
\hline 700 & 0.0022147 \\
\hline 750 & 0.00052118 \\
\hline Internal & \\
\hline 500 & 113.150 \\
\hline 550 & 8.5714 \\
\hline 600 & 0.83871 \\
\hline 650 & 0.051443 \\
\hline 700 & 0.0030569 \\
\hline 750 & 0.00059243 \\
\hline
\end{tabular}




\subsubsection{Pure External \& Internal Conversion, Out of time}

For out of time protons we see the same result, RPC signals are simulated to occur at a rate that is not of concern to Mu2e. The data table below shows that the amount of signal generated through RPC is well below one throughout the entirety of the experiment. This gives confidence that background due to RPC is unlikely throughout the data collection period of Mu2e.

TABLE 5.2

Background from Out-of-Time Protons for External and Internal Conversion Processes

\begin{tabular}{|c|c|}
\hline Time (ns) & Signals \\
\hline External & \\
\hline 500 & 0.0088577 \\
\hline 550 & 0.00848912 \\
\hline 600 & 0.00811615 \\
\hline 650 & 0.00773503 \\
\hline 700 & 0.00736776 \\
\hline Internal & \\
\hline 500 & 0.0111097 \\
\hline 550 & 0.106346 \\
\hline 600 & 0.0101615 \\
\hline 650 & 0.00968261 \\
\hline 700 & 0.0092069 \\
\hline 750 & 0.00873032 \\
\hline
\end{tabular}




\subsubsection{Combined Final Results}

With a nominal live-gate start time of 700ns, we look to the combined number of false-positive signals generated from radiative pion capture. We sum our signal values at the 700ns mark and yield a value of 0.02184626, which means that there is an extremely low probability associated with RPC having an effect on the Mu2e study. One way to interpret this data is to see it as indicating a 2.2\% chance of RPC having an effect on our data collection throughout the three year lifetime of this experiment.

\subsection{Final Conclusions}

As stated above, the expected effect of RPC on the Mu2e experiment is negligible with a $2.2 \%$ chance of producing a false-positive signal throughout the three year lifetime of the experiment. However, an important caveat is that this study focused solely on the background due to RPC, and did not take other backgrounds into account during these simulations. These results are referred to as “pure”, as we focus only on RPC. In the summer of 2019 we will conduct RPC background simulations which will take other various forms of background into account. These results will be referred to as "mixed"; moreover, we estimate that these mixed results will also point to RPC having a minimal effect on the Mu2e experiment. 


\section{REFERENCES}

Atanov et al. The calorimeter of the Mu2e experiment at Fermilab. Journal of Instrumentation, Jan 2017.

J. Bistirlich, K. M. Crowe, A. S. L. Parsons, P. Skarek, and P. Truöl. Photon spectra from radiative absorptions of pions in nuclei. Physical Review C, 5:1867-1883, Jun 1972.

Ciampa, K. Jul 2018. Design of the Mu2e Straw Tracker [PDF] http://Iss.fnal.gov/archive/2018/slides/fermilab-slides-18-118-nd.pdf

Glenzinski D. Sep 2016. A Rare Opportunity, the Mu2e Experiment [PDF] http://mu2e-docdb.fnal.gov/cgi-bin/RetrieveFile?docid=8084\&filename=Mu2 e-Seminar-MUSE-2016.pdf\&version=1

D. J. Griffiths. Introduction to Elementary Particles. Wiley-VCH, 2008.

A. Ilakovac, A. Pilaftsis, and L. Popov. Charged Lepton Flavor Violation in Supersymmetric Low-Scale Seesaw Models. arXiv:1212.5939v4, Feb. 2013.

H. Nunokawa, et al. CP Violation and Neutrino Oscillations. 2008 Prog. Part. Nucl. Phys.

Particle Data Group. The Particle Adventure. URL http://www.particleadventure.org/standard\%5fmodel.html 
The Mu2e Collaboration. Mu2e Technical Design Report. arXiv:1501.05241v2, Mar. 2015. The primary source for the information in Ch. 3 .

The Mu2e Collaboration. Official Mu2e Collaboration List, 2016. 


\section{CURRICULUM VITAE}

NAME: Joseph Garrard Leibson

ADDRESS: Department of Physics

University of Louisville

Louisville, KY 40292

DOB: $\quad$ April 1, 1993

EDUCATION: B.S. Physics

University of Louisville

2016 


\section{WORK EXPERIENCE}

\section{University of Louisville}

Graduate Teaching Assistant, 2017-2019 Dept. of Physics \& Astronomy

\section{University of Louisville}

Graduate Research Assistant, 2017-2019 UofL HEP Group

University of Louisville, PRIMES Program

Undergraduate Teaching Assistant, 2014-2016 Dept. of Physics \& Astronomy

University of Louisville

Undergraduate Research Assistant, 2013-2016

UofL HEP Group

Dr. John Morrison Research Group 


\section{TEACHING EXPERIENCE}

University of Louisville

Calculus Based Physics Laboratory I (Physics 295)

Algebra Based Physics Laboratory I (Physics 223)

Intro to Astronomy Laboratory (Physics 108) 